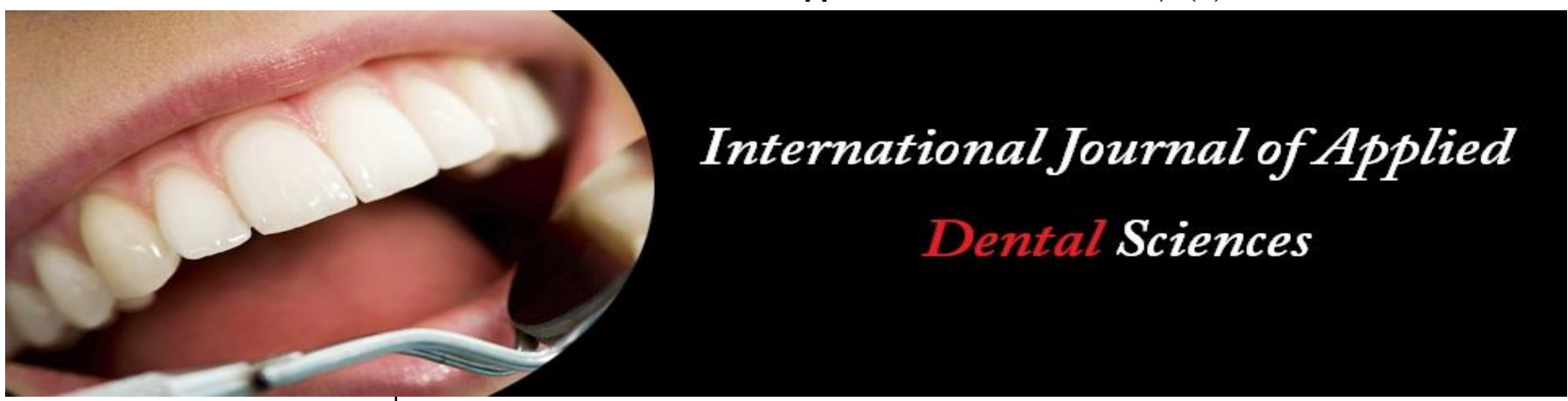

ISSN Print: 2394-7489

ISSN Online: 2394-7497

IJADS 2021; 7(4): 75-77

(C) 2021 IJADS

www.oraljournal.com

Received: 04-08-2021

Accepted: 06-09-2021

Ubaid Iqbal

Assistant Professor,

Department of Prosthodontics,

Government Dental College,

Srinagar, Jammu and Kashmir, India

Mohammad Arif Lone

Lecturer Department of

Prosthodontics, Government

Dental College, Srinagar,

Jammu and Kashmir, India

Shabir Ahmad Shah

Professor Department of

Prosthodontics, Government

Dental College, Srinagar,

Jammu and Kashmir, India

\section{Shazana Qazi}

Associate Professor,

Department of Prosthodontics,

Government Dental College,

Srinagar, Jammu and Kashmir, India
Corresponding Author:

Ubaid Iqbal

Assistant Professor,

Department of Prosthodontics,

Government Dental College,

Srinagar, Jammu and Kashmir, India

\section{Study of the impression techniques used for implant dentistry by different practitioners in Kashmir}

\author{
Ubaid Iqbal, Mohammad Arif Lone, Shabir Ahmad Shah and Shazana \\ Qazi
}

DOI: $\underline{\text { https://doi.org/10.22271/oral.2021.v7.i4b.1353 }}$

\section{Abstract}

Aim \& Objective: A questionnaire based study on the impression techniques used for implants by practioners.

Materials and Methods: Questionnaire was made \& distributed to dental practitioners and students of different colleges. Around a 100 doctors were chosen by random sampling, and Questionnaire was given to those who were willing to participate. This research focuses on impression materials and the various impression techniques that can be used in implant restorations with note on recent advances in implant impressions.

Results: $88.71 \%$ of the dentists feel that special components are required for an implant impression and majority believe that the level at which the impression should be taken depends on many factors. Majority believes that an FPD like impression is sufficient for cement retained FPD and also the fact that implant level impression can be taken for multiple angulated implants. Most of the dentists support the fact that multiple implants should be splinted before an impression and the most accepted material for an implant is addition silicone.

Keywords: questionnaire, osseointegration, polysiloxanes

\section{Introduction}

A dental implant is a fixture that is placed within bone that has been prepared for its insertion $\&$ the placement is a surgical procedure in which the implant interfaces with the bone to support a dental prosthesis like a crown or a bridge. Implant has now become a major part of general dental practice, however it requires a team of expertise who would help to achieve an aesthetically pleasing and a biocompatible restoration. Prosthodontic planning should be done beforehand the surgical procedure $\&$ is an essential part to arrive at a good prognosis for the dental implant ${ }^{[1]}$. The main goal of an implant impression is to accurately relate an implant or the abutment of the implant to other structures in the dental arch. Replicating the oral cavity and transferring it to a laboratory setting for implant fabrication is technique sensitive but it is essential for the success of an implant. In a implant impression, impression copings are utilized which is attached to the abutment or the implant. Various techniques have been suggested to achieve an accurate master cast. Open or transfer type and closed tray or pick up type are the most common techniques. In a pickup type of impression the coping is removed along with the impression whereas in a transfer type the coping is retained within the oral cavity. In some conditions, pick up type of preferable but it might be difficult to place the impression copings into the material correctly due to any reason. In a pickup type impression, rotation of the copings during fastening can cause a misfit ${ }^{[2]}$.

The osseointegration and the passive fit of the prosthesis determine the success of a dental implant. Osseointegration in itself is depended on implant design, surface, biocompatibility, and implant bed, surgical procedure. Implants lack the mobility of the periodontal ligament as it is ankylosed with the bone, the movement found in implant is maximum 5 micron ${ }^{[3]}$. Distortion or misfit in the implant abutment interface cannot be accommodated; Hence implant impression is one of the most important steps in achieving a passive fit by accuracy ${ }^{[4]}$. The main difficulty while coping with the impression restorations is correct transfer of the 
relationship of the implant to the master cast. Several alternatives exist while managing the influence in implant prosthodontics ${ }^{[5]}$. The implant analog or the abutments analog may be transferred to the secondary impression. In order to replicate the implant abutments in the master caste an impression should be taken. And in order to achieve a successful impression, the choice of impression material and the technique used is essential ${ }^{[6]}$. Dislodging of the components while pouring the impression and cast fracture should be prevented. The cast should resist fracture even when the prepared abutments are small and thus the impression material should be sufficiently flexible and rigid. The most commonly used impression materials in implant dentistry are vinyl polysiloxanes and polyether rubber base impression materials ${ }^{[7]}$. In general the impression material that use putty like heavy body component, which gives rigidity to the impression and less viscous was impression material are preferred to avoid incorporation of air bubbles. There are numerous studies done to investigate the accuracy of impression materials for implant impression and it was found that polyether and viny-polysiloxane impressions provided superior reproduction in comparison with other impression materials ${ }^{[8]}$. The most important property of an impression material is its wettability, which helps provide detail reproduction in wet oral surfaces, and also reproduces the details accurately with gypsum slurry ${ }^{[9]}$. As vinyl siloxane is a hydrophobic material manufactures have added extrinsic surfactants to improve its wettability both intra-orally as well as with gypsum slurry, thus making it hyrophilized Vinyl Polysilixone and similar to Polyether. Eleven studies compared the accuracy of polyether and vinyl polysiloxanes (VPS), and 10 of 11 reported no difference between the 2 materials ${ }^{[10]}$. Direct Impression techniques (Open tray, Pick Up): A custom or stock open tray with access to the impression coping screws is required, which exposes the coronal ends of the impression coping. Impression material is syringed around the impression coping and filled tray is then inserted into the mouth, ensuring that guide pin of the impression coping is visible and protrudes through the hole in the tray ${ }^{[11]}$. Impression copings are unscrewed and they are removed from the mouth together with the set impression. The implant analogues are connected to the copings using the same screw precautions to be taken are: radiographically confirmed seating of impression coping to the implant and use vinyl gloves when elastomeric impression material is used this technique can be used for single tooth restorations, multiunit restorations and implant over dentures for either cement retained or screw retained prosthesis ${ }^{[12]}$. An advantage of this technique is the dentist can confirm the laboratory preparation and contour of the provisional prosthesis to achieve the desired healing and soft tissue contour before final crown fabrication ${ }^{[13,14,15]}$

\section{Materials and Methods}

A standard questionnaire was distributed to dental practitioners and postgraduate students. Around 62 doctors were chosen by random sampling and given to those who are willing to participate. This research focuses on impression materials and the various impression techniques that can be used in implant restorations.

\section{Results}

1. Do you require special components to make an ideal implant impression? This was answered by an overwhelming $88.71 \%$ who responded with a yes, $9.68 \%$ responded with a no and rest was not sure.

2. At what level do you think implant impression should be made? 20\% answered "at the implant level", $9.68 \%$ responded "at the abutment level" and a majority $69.60 \%$ responded by saying that it depended on many factors.

3. "Do you think that in an ideal patient with single missing tooth scenario where adequate bone volume and soft tissue are present, a conventional FPD like impression will be sufficient. $19.35 \%$ responded by saying that it is always sufficient. $35.48 \%$ responded by mentioning that it is never sufficient and the rest $45.16 \%$ responded by "sufficient for cemented crowns and not sufficient for screw retained crowns".

4. "For multiple angulated implants is it possible to make implant level impressions? $53.23 \%$ replied in the affirmative, $16.13 \%$ replied in the negative and the rest $30.65 \%$ responded by saying not sure.

5. Which type of impression component requires an open tray for making implant impressions? Was the next question? $49.18 \%$ opted for the Transfer/ conical type, $39.34 \%$ opted for the square/pickup type and $11.48 \%$ opted for the snap on type.

6. "Do you think splinting of impression components is necessary for multiple implant scenario?" was the next question. Majority of the surveyed Dentists, $80.93 \%$ replied in the affirmative, $11.48 \%$ said no and the rest $8.30 \%$ were unsure.

7. What materials do you think is not acceptable for splinting? a) Pattern resin b) self-cure acrylic resin c) light activated composite resin d) Pattern resin with floss or steel wires" was the next poser. $14.52 \%$ favored A and D, $40.32 \%$ favored B and C, $45.16 \%$ favored B only.

8. "In case verification jig is not passive, what should you do? Was the next query. $72.13 \%$ responded by saying "Make a new pick u pick impression after sectioning and re-joining the jig", $19.67 \%$ responded by saying "Make another non splinted impression" and $8.20 \%$ responded by saying

9. Which of the following impression material is not acceptable for implant impressions? To this question, the respondents had the following reply. $11.29 \%$ opted for additional silicone, $50 \%$ responded by condensation silicone and the rest $38.71 \%$ opted for polyether.

10. What consistency of impression material is not appropriate for implant impressions? Was the last question? 64.52\% opted for light body, 29.03\% opted for Medium body/ monophase and $6.45 \%$ opted for Putty with light body.

\section{Discussion}

Oral rehabilitation with an implant-supported prosthesis has been well documented in the dental literature. This therapy is widely used in the dental clinics, and there is a growing demand from clinicians and patients to optimize the treatment protocols. Since the discovery of osseointegration, changes in implant-design, surface configuration, surgical techniques, restorative modalities and improvements in diagnostic techniques and pre-surgical planning tools has occurred. Change in technology and material advancement has tremendous commercial impact for the patients and dentists This survey was carried out to analyze the most preferred techniques and materials used by the dentists in an implant retained restorations across the world. In the study, it was evidently visible that there was a large discrepancy among the dental practitioners pertaining to the level at which the 
impression had to be taken. Though a large majority believed that it was associated with multiple factors, the participants had conflicting ideas as to whether it was at the level of the abutment $(9.68 \%)$ or the implant $(27.42 \%)$. Other participants said that the level at which the impression should be made depends on many factors. In a cemented type of an implant since the abutment projects supra-gingivally just like the abutment tooth of an FPD, a regular FPD impression is sufficient to record the finer details of the abutment and hence assists with good recreatibility of the abutment providing a better fit on cementing the crown. However in the screw retained type of abutment since it is located subgingivally and the crown is fixed onto it with the help of a screw the fixed dental prosthesis impression will not be helpful in recording the abutment. $45.16 \%$ of the participants believe that an FPD like impression is sufficient for cement retained implants and $35.48 \%$ of the participants believe that it is never sufficient. Multiple angulated implants affect the accuracy of the implant impression. $53.23 \%$ of the participants believe that implants level impressions can be made in case of multiple angulated implants. $16.13 \%$ of the participants seem to be sure that it is not possible and $30.65 \%$ of the people were not sure. Majority of the dental practitioners believe that the open type tray was required for a snap on type (11.48\%) and pick up /square type $(39.34 \%)$. The open type tray serves the function of the conical extension found on the transfer type of abutments. If the requirement of the opening is to provide room of this extension whereas there are no overextending structures in either the pick up or the snap on type. This was one of the misconceptions noticed among the practitioners. The study shows that the dentists here believe that special components such as impression copings play and important role in implant recording where others say most of the practitioners there didn't require the need for any special components for implant recording. This can be associated with the type of the implant materials used there and the difference in the technique for impressions taking by the dentists also. In regard to splinting for multiple implant impressions, a majority of the dental practitioners here (80.33) believed that splinting was required in order to ensure a good and stable impression, This relates either to the quality of impression taking skill or the type of the implant and the coping used there. A good concordance was seen between the group of practitioners of this study and a study performed by Lee et al. showing that both of the population preferred using auto polymerizing (self cure acrylic resin) with only a mild variation in the correlated study where they preferred using dental floss along with the auto polymerizing resin. Polyether was the material of choice for making implant impressions by most of the dentists across the world, as mentioned by many studies done to find out an accuracy of impression materials. However in our study polyether was the second preferred impression materials by $38.71 \%$ of dentists. The most ideal impression material is a putty impression refined with a light body. The dental practitioners showed a good knowledge of this fact which was evident from the majority of $93.65 \%$ saying that medium body (monophase) and light body on their own were poor choices in obtaining a good and accurate impression, but a meager $6.45 \%$ completely contradicted this fact.

\section{Conclusion}

Within the limitation of the study few questions were framed on the practitioner's opinion, but not literature evidence. It can be concluded from the survey that, most of the dentists follow the well-documented technique and proven materials, which have been documented in the literature and in an evidenced based practice, thus delivering the best to their patients. However, it is not necessary that every Implantologist should follow the protocol that is advice, every dentist have his or her way of attaining perfect impression.

\section{References}

1. Khalifa N, Allen PF, Abu-bakr NH, Abdel-Rahman ME. Factors associated with tooth loss and prosthodontic status among Sudanese adults. J Oral Sci 2012;54:303312. doi: 10.2334/josnusd.54.303.

2. Oliver RC, Brown LJ. Periodontal diseases and tooth loss. Periodontol 2000. 1993;2:117-127. doi: 10.1111/j.1600-0757.1993.tb00224.x.

3. Hollister MC, Weintraub JA. The association of oral status with systemic health, quality of life, and economic productivity. J Dent Educ 1993;57:901-912.

4. Ashley ET, Covington LL, Bishop BG, Breault LG. Ailing and failing endosseous dental implants: a literature review. J Contemp Dent Pract 2003;15:35-50.

5. Sorrentino R, Gherlone EF, Calesini G, Zarone F. Effect of implant angulation, connection length, and impression material on the dimensional accuracy of implant impressions: an in vitro comparative study. Clin Implant Dent Relat Res 2010;12(Suppl 1):e63-e76.

6. Lee H, Ercoli C, Funkenbusch PD, Feng C. Effect of subgingival depth of implant placement on the dimensional accuracy of the implant impression: an in vitro study. J Prosthet Dent 2008;99:107-113. doi: 10.1016/S0022-3913(08)60026-8

7. Baig MR. Multi-unit implant impression accuracy: a review of the literature. Quintessence Int 2014;45:39-51.

8. Osman MS, Ziada HM, Abubakr NH, Suliman AM. Implant impression accuracy of parallel and non-parallel implants: a comparative in-vitro analysis of open and closed tray techniques. Int J Implant Dent 2019;19(5):210. doi: 10.1186/s40729-019-0159-5.

9. Stimmelmayr M, Guth JF, Erdelt K, Happe A, Schlee M, Beuer F. Clinical study evaluating the discrepancy of two different impression techniques of four implants in an edentulous jaw. Clin Oral Investig 2013;17:1929-1935. doi: $10.1007 / \mathrm{s} 00784-012-0885-\mathrm{z}$.

10. SA New classification of physical status. Anesthesiology 1963;24:111. D.G.

11. Atsumi M, Park SH, Wang HL. Methods used to assess implant stability: current status. Int J Oral Maxillofac Implants 2007;22:743-754.

12. Martin W, Lewis E, Nicol A. Local risk factors for implant therapy. Int $\mathbf{J}$ Oral Maxillofac Implants 2009;24(Suppl):28-38.

13. Sethi A, Kaus T, Sochor P. The use of angulated abutments in implant dentistry: five-year clinical results of an ongoing prospective study. Int J Oral Maxillofac Implants 2000;15:801-810.

14. Lee SJ, Gallucci GO. Digital vs. conventional implant impressions: efficiency outcomes. Clin Oral Implants Res 2013;24:111-115. doi: 10.1111/j.1600-0501.2012.02430.x

15. Kan JY, Rungcharassaeng K, Bohsali K, Goodacre CJ, Lang BR. Clinical methods for evaluating implant framework fit. J Prosthet Dent 1999;81:7-13. doi: 10.1016/S0022-3913(99)70229-5 